\title{
Kinetics of Circulating Plasma Cell-Free DNA in Paediatric Classical Hodgkin Lymphoma
}

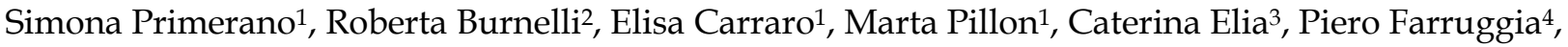 \\ Alessandra Sala ${ }^{5}$, Luciana Vinti ${ }^{6}$, Salvatore Buffardi 7 , Giuseppe Basso ${ }^{1}$, Maurizio Mascarin ${ }^{3 *}$ and Lara \\ Mussolin $1,8^{* 凶}$ \\ 1. Clinica di Oncoematologia Pediatrica, Dipartimento Salute della Donna e del Bambino, Azienda Ospedaliera-Universita' di Padova, Padova; \\ 2. Unita' di Oncoematologia, Azienda Ospedaliero-Universitaria di Ferrara S.Anna, Ferrara; \\ 3. SOS di Radioterapia Pediatrica CRO Aviano; \\ 4. Dipartimento di Oncologia, Unita' di Oncoematologia Pediatrica, A.R.N.A.S. Ospedali Civico di Cristina e Benfratelli, Palermo; \\ 5. Clinica Pediatrica, Università di Milano-Bicocca, Ospedale S.Gerardo, Fondazione Monza e Brianza per il Bambino e la sua Mamma, Monza; \\ 6. Dipartimento di Oncoematologia Pediatrica, Ospedale Bambino Gesu', Roma; \\ 7. Dipartimento di Oncologia Pediatrica, Ospedale Santobono-Pausillipon, Napoli; \\ 8. Istituto di Ricerca Pediatrica-Fondazione Città della Speranza, Padova. \\ ${ }^{*}$ co-last authors \\ $\triangle$ Corresponding author: Lara Mussolin, PhD. Laboratorio Biologia Tumori Solidi - Istituto di Ricerca Pediatrica- Fondazione Città della Speranza, Clinica di \\ Oncoematologia Pediatrica, Dipartimento della Salute della Donna e del Bambino, Universita' degli Studi di Padova, Corso Stati Uniti, 4- 35128 Padova (Italy).
}

phone: +39 049 8215565; fax: +39 049 9640150; e-mail: lara.mussolin@unipd.it

(C) Ivyspring International Publisher. Reproduction is permitted for personal, noncommercial use, provided that the article is in whole, unmodified, and properly cited. See http://ivyspring.com/terms for terms and conditions.

Received: 2015.08.18; Accepted: 2015.12.08; Published: 2016.01.12

\begin{abstract}
Levels of plasma cell-free DNA (cfDNA) of a large series of children with classical Hodgkin lymphoma ( $\mathrm{cHL}$ ) were evaluated and analyzed at diagnosis and during chemotherapy treatment in relation with clinical characteristics. CfDNA levels in $\mathrm{cHL}$ patients were significantly higher compared with controls $(p=0.002)$. CfDNA at diagnosis was correlated with presence of $B$ symptoms $(p=0.027)$ and high erythrocyte sedimentation rate $(p=0.049)$. We found that the increasing of plasma cfDNA after first chemotherapy cycle seems to be associated with a worse prognosis $(p=0.049)$. Levels of plasma cfDNA might constitute an interesting non-invasive tool in cHL patients' management.
\end{abstract}

Key words: classical Hodgkin Lymphoma, cell-free DNA, childhood.

\section{Introduction}

Classical Hodgkin lymphoma (cHL) is a malignant disease of the lymphatic system, histologically characterized by multinuclear Hodgkin's and Reed-Sternberg cells, that usually account for less than $1 \%$ of cells in the tumour tissue and are embedded in a reactive infiltrate. Clinical trials have the goal of maintaining high cure rates while minimizing late effects by identification of patients who are most likely to benefit from reduced-intensity therapy. For this reason identification of biological parameters for monitoring disease during therapy and follow-up should be strongly required.

\section{Materials and Methods}

Peripheral blood samples of paediatric HL patients were collected from Associazione Italiana di Emato-Oncologia Pediatrica (A.I.E.O.P.) centers in the laboratory of Padua University in the period from December 1, 2004 to August, 31 2014. We evaluated cfDNA in plasma samples obtained at diagnosis (time point TP0) in a total of 155 cases: in $75 / 155$ we also analyzed time-point TP1 (after the first chemotherapy cycle), in 41/155 TP2 (after stop chemotherapy) and in 25/155 cases TP3 (after radiotherapy, if required by protocol). 
Written consent was obtained from parents or legal guardians of each patient before enrolment. The study was approved by the ethics committee of each participating Institution. A control group of 15 pediatric healthy individuals ( 7 males, 8 females; median 12 yrs, range 5-16) was included to determine the normal range of circulating cfDNA in plasma. Peripheral blood samples in sodium citrate were processed within 24 hours from drawing and plasma cfDNA was quantified using a Taq-Man based real-time PCR assay for the POLR2 gene as previously reported [1]. Wilcoxon rank sum test was used to compare plasma levels between different patient subgroups and controls. Associations of cfDNA with specific clinical characteristics were tested with Chi-square test and Fisher's exact test when appropriate.

\section{Results}

From 2004, in Italy, children and adolescents with cHL were enrolled in a multicenter clinical trial of A.I.E.O.P, namely AIEOP LH-2004. Patients, staged according to Ann Arbor classification [2], were allocated to three different therapeutic groups, based on risk groups. In the present study, we investigated the presence of circulating cell-free DNA (cfDNA) in plasma at diagnosis and during chemotherapy of a large series of pediatric cHL patients and analyzed its association with clinical features. As cancer cells grow, nucleic acids are released into the bloodstream through apoptosis, necrosis or secretion $[3,4]$. Nucleid acids can be found also in small amounts in plasma of healthy individuals, however blood levels of cfDNA in plasma of patients with tumour are usually $2-3$ times higher than in normal healthy subjects [5].

The median age at diagnosis for $155 \mathrm{cHL}$ patients was 13.6 years (range 2.7-19.7) and 51\% (79/155) were females. The 2-year event free survival ( \pm standard error, SE) and overall survival were $83 \%$ ( \pm SE $4 \%$ ) and $98 \%( \pm$ SE 2\%) respectively. Patients who achieved clinical remission (complete or partial remission $>75 \%$ ) were 134, while 21 patients had a relapse or a progression (relapse was considered pathologically-confirmed recurrence of $\mathrm{cHL}$, and progression the same condition diagnosed within 3 months of the end of therapy) at the median time of 0.85 year (range 0.28-5.8 years) from diagnosis. Three patients out of 155 died of progressive disease.

The concentration of cfDNA in plasma samples at TP0 was significantly higher than in the controls (mean value $112 \mathrm{ng} / \mathrm{mL}$ in cHL cases versus 5.5 $\mathrm{ng} / \mathrm{mL}$ in healthy controls, $p=0.002$ ). We found that high cfDNA plasma concentrations at diagnosis correlated with presence of B symptoms $(p=0.027)$ and high erythrocyte sedimentation rate $(\mathrm{ESR})(p=0.049)$.
No association was found between cfDNA levels and other clinical characteristics analyzed (gender, median age, stage, therapeutic group, bulky disease). Considering that B-symptoms are due to the production of pro-inflammatory cytokines by the Hodgkin tumor tissue and ESR is a marker of inflammatory reaction, we could speculate that in cHL patients high levels of cfDNA correlate with inflammatory status and disease activity. As observed in patients with colorectal cancer, most of cfDNA did not derive from the neoplastic cells themselves, but from engulfment by macrophages of neoplastic cells and surrounding stromal and inflammatory cells within the tumour [6]. This is particular interesting if we consider that HL is characterized by only a small fraction of tumor cells surrounded by a massive inflammatory infiltrate.

Plasma cfDNA levels at time points TP1, TP2 and TP3 were also quantified in an unselected subgroup of our cHL cohort to evaluate the kinetics of this biological marker. Overall, a decrease of median cfDNA level was observed during chemotherapy treatment $(\mathrm{TP} 0=40 \mathrm{ng} / \mathrm{mL} ; \mathrm{TP} 1=22 \mathrm{ng} / \mathrm{mL} ; \mathrm{TP} 2=22$ $\mathrm{ng} / \mathrm{mL} ; \mathrm{TP} 3=15 \mathrm{ng} / \mathrm{mL}$ ). Of note, cfDNA increased of at least 1.5 times compared with diagnosis at TP1 in $24 / 75$ patients $(32 \%)$ and we observed a significant correlation with mediastinal bulky involvement $(p=0.03)$. In particular, 40/75 (53\%) patients presented mediastinal bulky disease at diagnosis and in $45 \%$ $(18 / 40)$ there was an increasing of cfDNA at TP1 whereas, in the remaining 35/75 (47\%) patients without mediastinal bulky disease at diagnosis, only $17 \%(6 / 35)$ had a cfDNA increasing at TP1. We evaluated whether cases with increasing of cfDNA between the different time points (TP0 vs TP1; TP0 vs TP2; TP0 vs TP3; TP1 vs TP2; TP1 vs TP3; TP2 vs TP3) were associated to relapse/progression and we found that the increasing of cfDNA in TP1 compared to TP0 was significantly associated to relapse/progression $(p=0.049)$ (Fig.1). In particular, there was $20 \%$ of events in the subgroup of 24 patients with increasing cfDNA at TP1, versus $8 \%$ of events in the subgroup of 51 patients with decreasing cfDNA at TP1 compared to TP0.

\section{Discussion}

Tumour specific molecular markers are important clinical tools for disease monitoring; in cHL the absence of these markers has always prevented this kind of investigation. Circulating cfDNA could not still be used as independent prognostic factor in cHL but, based on our data, it has to be considered for further analysis. Our study showed that in cHL of childhood and adolescence circulating plasma cfDNA at diagnosis correlate with a diffuse inflammatory status. Recently in adult cHL, Romano et al.[7] 
showed that circulating myeloid-derived suppressor cells (MDSC) are increased at diagnosis and could predict short progression free survival. The accumulation of MDSC is a hallmark of malignancy-associated inflammation and $\mathrm{T}$ cell suppression in cancer [8]. However, the authors underlined that the use of MDSC as single biomarker is not sufficient because chronic inflammation status can trigger MDSC expansion and the intensive hematopoiesis during chemotherapy can favor MDSC release limiting their usage during treatment. In the next future, it could be very interesting to analyze, in pediatric patients, MDSC levels together with circulating cfDNA to evaluate if these two tumor inflammation markers are independent and could be used together in a risk-adapted therapeutic strategy.We found that the increasing of cfDNA after first chemotherapy cycle seems to be associated with a worse prognosis. To identify reliable parameters to predict the outcome in the single patient remains a challenge in pediatric oncology, but cfDNA study could play a significant role in this challenge for the next clinical trials in cHL.

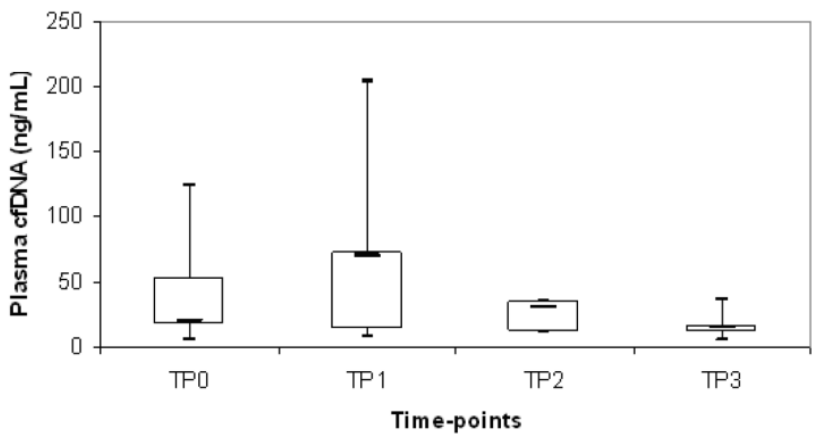

Figure 1. Distribution of plasma cfDNA during chemotherapy treatment in $\mathrm{cHL}$ patients who experienced relapse or progressive disease. The upper border of the box indicated the upper quartile $\left(75^{\text {th }}\right.$ percentile) while the lower border indicate the lower quartile $\left(25^{\text {th }}\right.$ percentile) and the horizontal line in the box the median. TPO= diagnosis; TP1=after $1^{\text {st }}$ chemotherapy cycle; TP2= stop chemotherapy; TP3=after radiotherapy.

\section{Competing Interests}

The authors have declared that no competing interest exists.

\section{References}

1. Mussolin L, Burnelli R, Pillon M, Carraro E, Farruggia P, Todesco A, Mascarin M, Rosolen A. Plasma Cell-free DNA in Paediatric Lymphomas. J Cancer 2013;4:323-329.

2. Carbone PP, Kaplan HS, Musshoff K, Smithers DW, Tubiana M. Report of the Committee on Hodgkin's Disease Staging Classification. Cancer Res. 1971 Nov;31(11):1860-1.

3. Stroun M, Lyautey J, Lederrey C, Olson-Sand A, Anker P. About the possible origin and mechanism of circulating DNA apoptosis and active DNA release. Clin Chim Acta 2001;313:139-142.

4. Stroun M, Lyautey J, Lederrey C, Mulcahy HE, Anker P. Alu repeat sequences are present in increased proportions compared to a unique gene in plasma/serum DNA: evidence for a preferential release from viable cells? Ann N Y Acad Sci 2001;945:258-264.

5. Fleischhacker M, Schmidt B. Circulating nucleic acids (CNAs) and cancer--a survey. Biochim Biophys Acta 2007;1775:181-232.
6. Diehl F, Li M, Dressman D, He Y, Shen D, Szabo S, Diaz LA Jr, Goodman SN, David KA, Juhl H, Kinzler KW, Vogelstein B. Detection and quantification of mutations in the plasma of patients with colorectal tumors. Proc Natl Acad Sci U S A. 2005;102:16368-16373.

7. Romano A, Parrinello NL, Vetro C, Forte S, Chiarenza A, Figuera A, Motta G, Palumbo GA, Ippolito M, Consoli U, Di Raimondo F. Circulating myeloid-derived suppressor cells correlate with clinical outcome in Hodgkin Lymphoma patients treated up-front with a risk-adapted strategy. Br J Haematol 2015;168:689-700.

8. Gabrilovich D, Ostrand-Rosenberg S, Bronte V. Coordinated regulation of myeloid cells by tumours. N Nat Rev Immunol 2012;12:253-268. 